Article

\title{
Speckle Noise Reduction in Digital Holography Using a DMD and Multi-Hologram Resampling
}

\author{
John Restrepo (D), Nelson Correa-Rojas *(D) and Jorge Herrera-Ramirez *(D) \\ Facultad de Ingeniería, Departamento de Electrónica y Telecomunicaciones, Instituto Tecnológico Metropolitano, \\ Medellín Calle 54A 30-01, Colombia; johnrestrepo8852@correo.itm.edu.co \\ * Correspondence: nelsoncorrea@itm.edu.co (N.C.-R.); jorgeherrerar@itm.edu.co (J.H.-R.)
}

Received: 26 September 2020; Accepted: 31 October 2020; Published: 22 November 2020

\begin{abstract}
Speckle noise is a well-documented problem on coherent imaging techniques like Digital Holography. A method to reduce the speckle noise level is presented, based on introducing a Digital Micromirror Device to phase modulate the illumination over the object. Multiple holograms with varying illuminations are recorded and the reconstructed intensities are averaged to obtain a final improved image. A simple numerical resampling scheme is proposed to further improve noise reduction. The obtained results demonstrate the effectiveness of the hybrid approach.
\end{abstract}

Keywords: digital holography; digital micromirror device; speckle; noise reduction

\section{Introduction}

Robust and versatile imaging techniques are always at the forefront of development in engineering and sciences. Digital Holography $(\mathrm{DH})$ provides an interesting alternative as it allows retrieving the three-dimensional information from a scene. Its potential has given rise to different applications in imaging objects [1], microscopy [2], quantitative phase estimation [3], and many others. Using coherent illumination, a holographic register is made by the light interference of the reference beam and object beam coming from the transmission or reflection of an object. The holograms are recorded digitally to be numerically reconstructed, retrieving the amplitude and phase information. When coherent light strikes an object with rough surfaces scaled to the visible wavelength range, a coherent noise or speckle appears. The hologram stores this noise along with the object information, and its presence extends to the reconstructed image deteriorating its quality.

Multiple strategies to reduce the speckle noise on DH have been proposed. They can be categorized, according to the number of holograms registered, into multiple and single-shot strategies. According to their approach, they can be divided into two categories: optical and numerical operations. Optical techniques tend to require multiple registers, as a specific property is modified between measurements to produce uncorrelated noise. Some examples of existing strategies include recording holograms with different wavelengths [4], polarization states [5], rotating diffuser [6], lateral shifting of the camera [7], lateral shifting of the object [8], and time multiplexing [9]. The multiple registers are expected to have a differently behaved noise, which implies that a simple averaging operation can produce a new intensity image with less coherent noise. The drawbacks inherent for these techniques come from the complexity of the optical set-ups and the inability to register dynamic events provided multiple registers are needed.

Numerical techniques, on the other hand, tend to achieve the noise reduction based on a single hologram. There are approaches with traditional image processing techniques $[10,11]$ where the reconstructed intensity is processed to improve its quality. Other techniques try to obtain multiple reconstructions from a single hologram, by rectangular segment subdivision [12] or grid-like subdivision [13] of the recorded hologram. These generated registers are consequently reconstructed 
and the resulting intensities averaged. As the resolution of a holographic reconstruction is affected by the size of the hologram, these techniques suffer from an increasing loss of resolution when increasing the subdivisions. Random non-Bayesian complementary masks have been proposed [14], where a multiplication with the original hologram produces multiple new registers with an expected different noise. The randomness of the sampling consequently affects the resolution of the reconstructed image.

In this paper, we propose a combination of a numerical and optical approach to reduce the speckle noise. We introduce a Digital Micromirror Device (DMD) into the holographic set-up that allows varying the phase of the illumination hitting the object. Multiple holograms are recorded with varying illumination. Similar techniques have been proposed in the past, albeit based on physically rotating a mirror [15,16] or a spatial light modulator [17]. Here, we propose the use of a DMD which is known to be a more robust and experimentally approachable device that offers a high speed of operation as it has been documented in the past [18] with its adaptation to a holographic setup. This feature allows us to implement synchronous registering with the camera at high frame rates reducing the constraints of this approach for dynamic applications. Furthermore, we present a numerical resampling of the recorded holograms, producing new synthetic holograms with more varying coherent noise. The combination of numerical and optical techniques has been attempted in the past under different methodologies [17,19], albeit with more complex processing algorithms. We evaluated our technique with different illumination phase patterns and demonstrate that the reduction in the speckle noise is significant.

\section{Digital Holography with a DMD}

\subsection{Digital Holography}

In a traditional digital holographic process, light emanating from a diffuse object interferes with a reference wave, forming the hologram over the sensor plane of a digital recording device. The two light beams, object and reference, are directed to have an oblique angle between them. The object reconstruction is obtained from the recorded discrete hologram $H(k, l)$ using a discrete Fresnel transform [1], given by

$$
\begin{aligned}
\Gamma(m, n)= & \frac{i}{\lambda d} \exp \left[-i \pi \lambda d\left(\frac{m^{2}}{N^{2} \Delta x^{2}}+\frac{n^{2}}{N^{2} \Delta y^{2}}\right)\right] \\
& \times \sum_{k=0}^{N-1} \sum_{l=0}^{N-1} R(k, l) H(k, l) \exp \left[-i \frac{\pi}{\lambda d}\left(k^{2} \Delta x^{2}+l^{2} \Delta y^{2}\right)\right] \\
& \times \exp \left[i 2 \pi\left(\frac{k m}{N}+\frac{l n}{N}\right)\right],
\end{aligned}
$$

where $\Delta x, \Delta y$ represent the pixel sizes, $N \times N$ the number of pixels, $\lambda$ the light wavelength, $d$ is the object distance to the hologram plane, $R$ is the reference complex wave, and $\Gamma$ is the reconstructed complex wave.

\subsection{Digital Micro-Mirror Device}

A Digital micromirror Device (DMD) is a versatile mechanism for passive light manipulation. It offers attractive experimental characteristics such as fast switching of the modulated signal, direct use avoiding any calibration, low spectrum sensitivity, and polarization invariance. Its main principle is to control the way light reflects on it through millions of small switchable mirrors that can be electronically set to two specific orientations [20]. The resultant operation is that of a multiplication of the incoming wave by a binary transmittance function composed by the lateral aggregation of all micromirrors, resulting in an amplitude-only control. However, a Lee Hologram [21] can be used to modulate phase and amplitude using a DMD as well. This Lee hologram is projected on the DMD and the emanating light is transferred to a Fourier filtering scheme, where by selecting the appropriate diffraction order 
the desired wave modulation is obtained. Lee [21] proposed to generate such hologram using the following expression [20],

$$
L(x, y)=\frac{1}{2}+\frac{1}{2} \operatorname{sgn}\left\{\cos \left[2 \pi x v_{x}-\phi(x, y)\right]+\cos [\pi \omega(x, y)]\right\},
$$

where $v_{x}$ is the carrier frequency, and $\omega(x, y)$ is a function related to the target complex wave $A(x, y) \exp (i \phi(x, y))$ by

$$
\omega(x, y)=\frac{1}{\pi} \arcsin [A(x, y)]
$$

Therefore, any desired complex wave can be obtained by designing the functions $\phi$ and $\omega$, and selecting an appropriate carrier spatial frequency. We consider the quality of this phase modulation technique to be sufficient for our application, despite the diffraction produced by the periodicity of the DMD and the diffraction noise inherent to the binary nature of the Lee holograms, as the Fourier filtering effectively isolates the desired modulated light beam [22].

Figure 1 shows our optical set-up. Light from a blue laser $\lambda=473 \mathrm{~nm}$ is split into the object and reference arms necessary for the holographic register. The object arm is directed towards the functioning DMD. The DMD diffracts this wave in multiple diffraction orders. One of those is inputted into a Fourier filtering system producing a single complex wave with a phase function designed according to the pattern displayed on the DMD. This filtered wave strikes the reflective object creating the object wave for the holographic recording. The camera records the combination of the object and reference waves, namely, the hologram, for subsequent digital reconstruction.

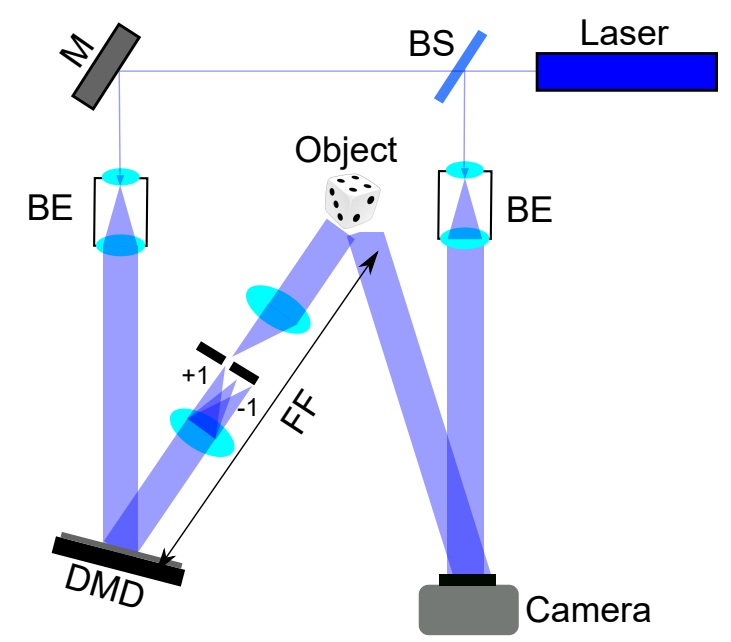

Figure 1. Digital holographic recording setup with a Digital Micromirror (DMD) for phase modulation. BE: Beam Expander, BS: Beam Splitter, DMD: Digital Micromirror Device, M:Mirror, FF: Fourier Filtering.

\section{Optical Speckle Reduction}

We evaluated two different methods for changing the illumination over the object. The first consists of using the DMD to illuminate the object with plane waves with varying tilt; the second method consists of illuminating the object with a random checkered phase function. Both strategies involve registering a hologram for each pattern on the DMD, producing multiple reconstructed images that are later averaged to reduce the speckle. The pipeline to generate a final intensity reconstruction with reduced speckle is shown in Figure 2. The holograms can be obtained with any of the techniques illustrated in the following sections. Each hologram is propagated with the discrete Fresnel Transform to obtain a reconstructed complex wave. This contains all the information stored in the hologram, the zero order located at the center and the separated twin images to its sides. The separation comes from the off-axis nature of the holographic recording. If the propagation distance $d$ is properly chosen, 
one of the images will be in perfect focus. From this image, we crop a square area containing the object, facilitating the further analysis of the reconstruction quality and its speckle. All the cropped intensity images are averaged and the result is a final reconstruction with reduced speckle noise.

These former strategies can be supported by characterizing the speckle as a multiplicative noise $[23,24]$. Thus, the result of the holographic reconstruction given in Equation (1) can be understood as the object field $E_{o} \exp \left(i \phi_{0}\right)$ modulated by a speckle noise $E_{s} \exp \left(i \phi_{s}\right)$ as follows [6],

$$
\Gamma=E_{o} \exp \left(i \phi_{o}\right) E_{s} \exp \left(i \phi_{s}\right)
$$

Likewise, the intensity of the reconstruction will be given by

$$
I=\Gamma \cdot \Gamma^{*}=E_{o}^{2} E_{s}^{2}
$$

where $\Gamma^{*}$ stands for the complex conjugate of $\Gamma$. Equation (5) allows us to continue interpreting the speckle $E_{s}^{2}$ as a multiplicative noise that modulates the distribution of intensities $E_{o}^{2}$ produced by the object. In this way, it can be observed that the intensity distribution of the object in the reconstruction does not change if only the phase characteristics of the object illumination are modulated between acquisitions. On the other hand, the intensity distribution of the speckle noise does change. This is because the phase modulation of the illuminating beam changes the phases at each point of the surface of the object, which affects the superposition of random phasors that gives rise to the intensity distribution of the speckle noise $[16,25]$.

Therefore, the superposition process of several hologram reconstructions with different speckle patterns can be written as

$$
\sum_{i=1}^{h} I_{i}=E_{o}^{2}\left(E_{s 1}^{2}+E_{s 2}^{2}+\ldots+E_{s h}^{2}\right)
$$

where $h$ is the number of superposed reconstructions. In Equation (6), we have a new speckle given by the superposition of intensities of $h$ different speckle patterns while preserving the information of the object. The contrast of the resulting speckle pattern is reduced by $1 / \sqrt{h}$ for the superposition of $h$ totally uncorrelated speckle patterns [25].

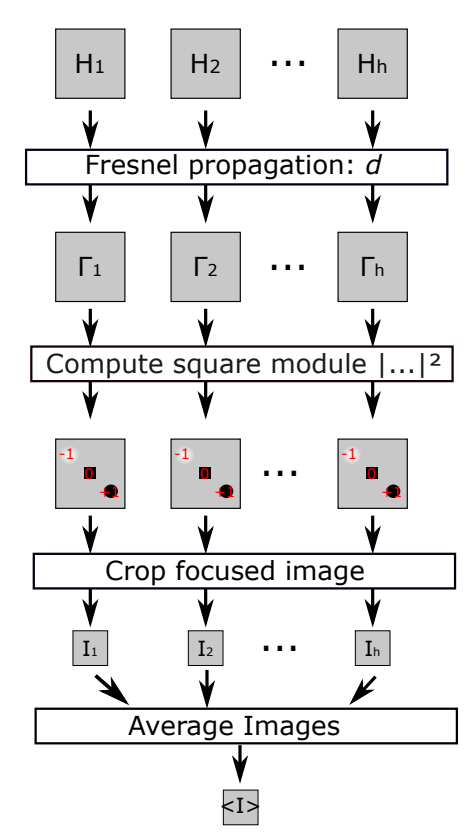

Figure 2. Pipeline for hologram reconstruction and speckle reduction. 


\subsection{Plane Wave Illumination}

The Lee hologram to generate a tilted plane wave is a set of binary linear fringes, which can be generated with the functions $\phi(x, y)=0$ and $A(x, y)=1$. The orientation of the fringes and their spatial frequency control the tilting of this plane wave. To establish the available range of tilt angles that can be used in our set-up, we considered that the angle of illumination produced by the DMD is related to the light and DMD pattern by [26]

$$
\theta_{X}=\sin ^{-1}\left(\frac{\lambda}{\Lambda_{X}}\right) \approx \frac{\lambda}{\Lambda_{X}}
$$

where $\lambda$ is the light wavelength, $\Lambda_{X}$ is the period of DMD pattern in the $X$ direction, and $\theta_{X}$ is the corresponding angle of the plane wave measured with respect to the optical axis. We implemented the Fourier filtering based on two positive lenses $f_{1}$ and $f_{2}$, and in this case, the angle in which a ray leaves the lens $f_{2}$ depends on the angle in which it enters the first $f_{1}$ by (under paraxial approximation)

$$
\theta_{X}^{\prime} \approx \frac{f_{1}}{f_{2}} \theta_{X}
$$

where $\theta_{X}^{\prime}$ is the output angle and $\theta_{X}$ is the input angle, both measured with respect to the optical axis.

Under these considerations, we have control over the angle of the plane wave that illuminates the object by simply changing the orientation and period $\Lambda$ of the binary pattern on the DMD, since the other parameters $\left(f_{1}, f_{2}, \lambda\right)$ can be considered as exclusively experimental factors and they are fixed for practical reasons. An example of a DMD pattern used to generate a plane wave in our experiments is displayed in Figure 3a, with experimental values $f_{1}=250 \mathrm{~mm}, f_{2}=500 \mathrm{~mm}, \lambda=473 \mathrm{~nm}$. We generated plane waves with an angular variation inside a range of $0.1 \mathrm{deg}$ for a total of $h=20$ recorded holograms.

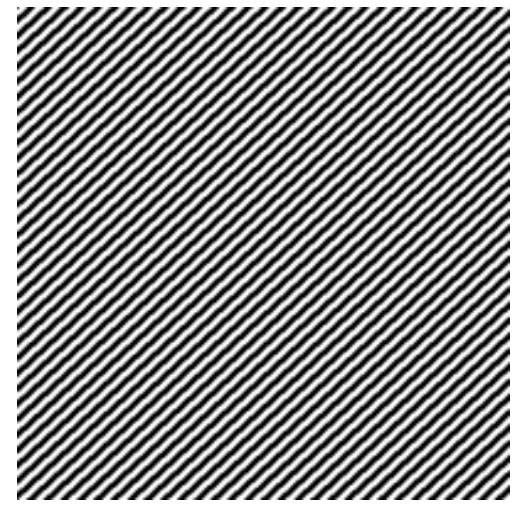

(a) Linear

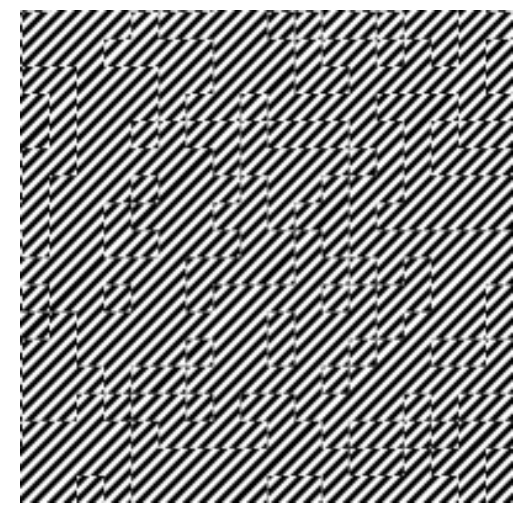

(b) Random checkered

Figure 3. (a) Square segment of the pattern for a plane wave illumination. (b) Square segment of the pattern for a randomly checkered phase modulated illumination.

\subsection{Random Checkered Phase Pattern}

The second method evaluated consists of displaying a binary, random sized checkered pattern on the DMD superposed to the binary fringes for a plane wave modulation. This DMD pattern is constructed with the function $\phi(x, y)$ being assigned a random checkered matrix containing 0 and $\pi$ values. The function $A(x, y)$ is again set to one to avoid amplitude modulation. The lateral displacement of the binary fringes, translates into pi radians jumps in the phase modulation, thus creating a randomly phase-modulated illumination.

Given the effect of the borders around each block element, there is a remainder of amplitude modulation on the illumination over the object, which is still visible on the reconstructed image. To diminish this effect, we project random patterns with differently sized block elements and consider 
only the combination of multiple reconstructions from different patterns. The averaging of the reconstruction helps in diminishing this unwanted amplitude effect. Figure $3 \mathrm{~b}$ presents a small region of a pattern of this kind. In here, the presence of the random checkered pattern can be observed, by noting the discontinuities in the fringes. The block elements are differently sized within the same pattern, and a new random distribution of the matrix assigned to $\phi(x, y)$ is computed to generate each new DMD pattern.

\section{Digital Holograms Resampling}

To complement the speckle reduction obtained from the multiple registered holograms, we studied a digital resampling scheme, where we generate several synthetic holograms from the original recorded ones. These new holograms are fabricated to increase the randomness of the speckle for each reconstructed image, thus allowing a larger speckle decrease in the final averaged image.

We interpret the set of $h$ holograms recorded as a 3D array, where a 2D slice corresponds to a single original hologram. The synthetic holograms are of identical size to the originals $N \times N$ and are generated from a random sampling of the full $3 \mathrm{D}$ volume. This sampling is done by multiplication with different random binary masks $M_{i}$ and the accumulation of the results as it is illustrated in Figure 4. The resulting synthetic hologram is composed of a series of patches that were contributed by the original holograms. The size of the patches $G$ and the matching of masks and holograms controls the level of randomness introduced on the synthetic hologram. With a smaller patch size, the binary masks become more complex, and the synthetic hologram less resembles any of the original. The number of masks required to generate a synthetic hologram is $b=(N / G)^{2}$. We compute the masks to maximize the number of recorded holograms that contribute. If $b=h$ then all holograms will always equally contribute to the generation of a synthetic one, what changes from one to the other is the location of the patches in the mask. Additionally for this case, every mask will only contain one patch. If $b<h$ then only a subset of the holograms will contribute to a new resampled one. To ensure maximum randomness, we use a selection without replacement from the set of $h$ holograms, so that no original is repeated. Again, each mask contains also a single a patch. An example of this case is illustrated in Figure 4 where $N=2048, G=512$ producing $b=16$. The synthetic hologram receives contributions of a subset of 16 holograms from the full set of $h=20$. To generate a new different synthetic, we randomize the location of the patches and select a new subset of holograms. If $b>h$ then the binary masks will contain multiple patches in them, as each synthetic hologram will have repeated contributions from the originals. Each mask $M_{i}$ is computed to have approximately the same number of patches, to ensure that no single original hologram is being preferred. This ensures a high level of randomness in the resampled holograms.

The patch size has to be designed to provide enough randomness and to utilize the full amount of recorded holograms $h$. However there are issues of loss of resolution for smaller values of $G$, which are discussed in a further section. In equation form, the synthetic hologram is obtained as

$$
\tilde{H}=\sum_{i}^{b} M_{i} H_{i}
$$

where the masks fulfill the sampling condition $\sum M_{i}=\mathbf{1}_{N \times N}$. Repeating the process and computing a new random distribution of masks generates more synthetic holograms, even surpassing the number of recorded holograms. 


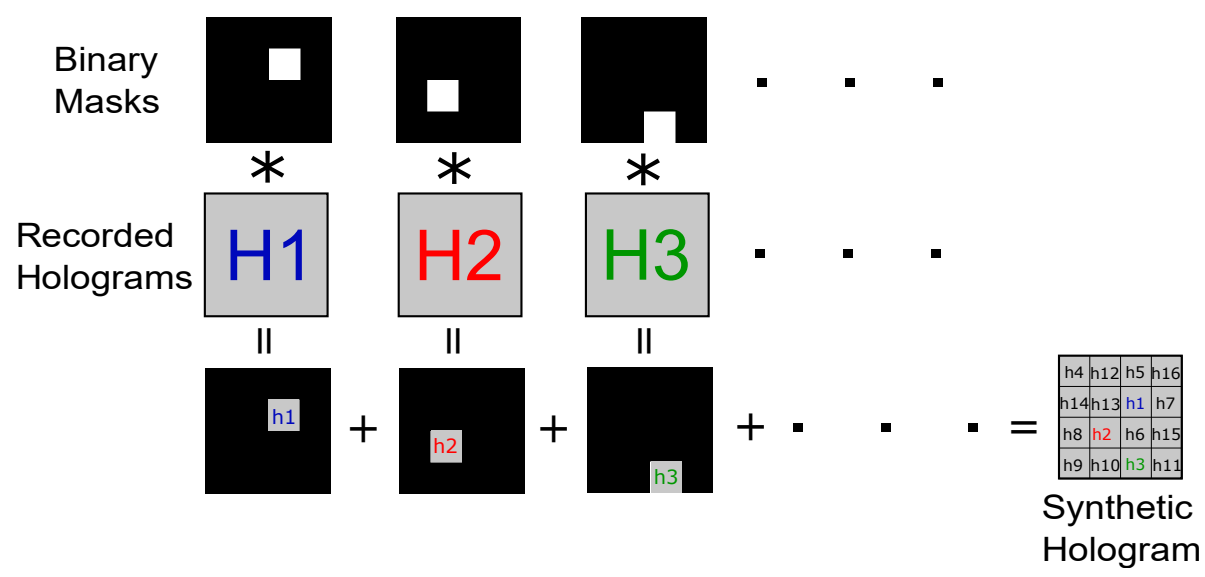

Figure 4. Digital resampling of the recorded holograms for $b=16, h=20$. Adding the individual Hadamard multiplications between holograms and binary masks produces a synthetic hologram. A hologram $\mathrm{H} 1$ contributes the small patch h1 to the final synthetic hologram.

\section{Speckle Reduction Evaluation}

We considered two metrics to quantify the level of speckle reduction. The speckle contrast coefficient $C$ and the correlation coefficient $\gamma$.

The speckle contrast coefficient is the most commonly used metric to have an understanding of the speckle level present in a reconstructed image [25]. The coefficient is computed by

$$
C=\frac{\sigma_{I}}{\langle I\rangle}
$$

where $\sigma_{I}$ represents the standard deviation and $\langle I\rangle$ the mean of the intensity values reconstructed. The evaluation of the coefficient is done over a homogeneous intensity region so that the changes on $C$ are due only to the speckle contribution. The coefficient follows a theoretical behavior of $1 / \sqrt{h}$ for $h$ reconstructed images [25], the lower its value is, the less speckle noise is present in the image.

As a second metric, we considered the correlation coefficient for a pair of reconstructed images $I_{t}$ and $I_{S}$ as an indication of the level of uncorrelation between them. The coefficient is obtained from the equation

$$
\gamma_{t, s}=\frac{\sum_{p}^{M} \sum_{q}^{L}\left[I_{t}(p, q)-\left\langle I_{t}\right\rangle\right]\left[I_{s}(p, q)-\left\langle I_{s}\right\rangle\right]}{\sqrt{\sum_{p}^{M} \sum_{q}^{L}\left[I_{t}(p, q)-\left\langle I_{t}\right\rangle\right]^{2} \sum_{p}^{M} \sum_{q}^{L}\left[I_{s}(p, q)-\left\langle I_{s}\right\rangle\right]^{2}}}
$$

The correlation coefficient is near a unit value for images with similar speckle distributions and near zero for uncorrelated images. Being an indicator of the relation between two images, we can compute a matrix of coefficients for the set of all registered holograms indicating which pairs of reconstructions are more uncorrelated.

\section{Results and Discussion}

\subsection{Recorded Holograms}

We separately evaluated our proposed strategies to decrease the level of speckle noise. The comparison of the results is summarized in Figure 5. For reference, we included reconstructed images from a single hologram in the first row, presenting the unavoidable speckle. In the second row, we show the resulting averaged images with decreased speckle. These images are the result of the average of 20 reconstructions. The improvement is qualitatively evident as shown by these images. More specifically, in Figure 6 we show a quantitative evaluation for comparing some results of the strategy using synthetic holograms, and we expand on the analysis of the results of this strategy in the next section. But regarding the two strategies using only recorded holograms, in the first two columns of Figure 7 we showcase the behavior of their speckle metrics. As is shown by the curves of the speckle 
contrast, the improvement is also quantitatively evident when more images are averaged. In the final row, the correlation coefficients for all reconstructed images are presented as a lower triangular matrix. On this plot, the indices of the matrix for the plane wave approach also denote an increment of the angle of tilt on the illuminating wave, so the color distribution in this matrix indicates that with more difference in the angles between plane waves, the correlation is smaller; specifically, the pair of images $(s=19, t=0)$ present the smallest value of $\gamma$, as we increase the value of the index $t$ keeping the value of $s$ constant, the two plane waves have gradually more similar tilts, producing an increase in the correlation coefficient. As such, these images will present similar speckle noise distributions. For the checkered random patterns, the correlation behaves randomly for any pair of images, as the computation of the checkered patterns introduces random changes. However given the conditions in our optical setup, the values obtained for $\gamma$ are not significantly better than the plane wave strategy. The diagonal in all matrices represents the result of computing the correlation of an image with itself, which results in a unitary value.

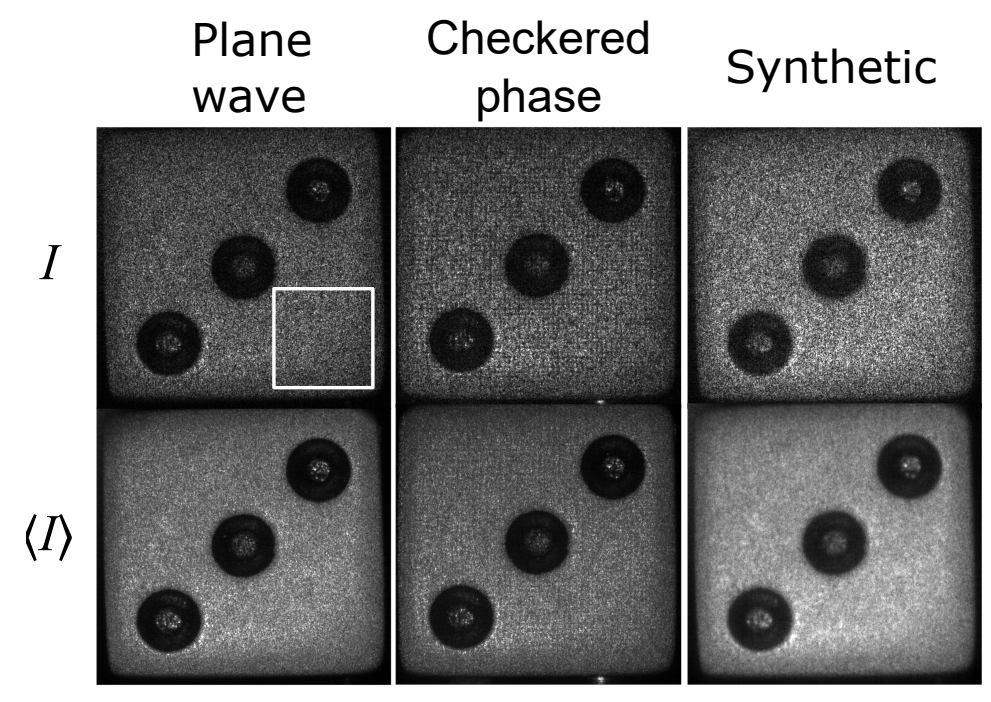

Figure 5. Results in reduction of speckle noise for the three methodologies. First row: single hologram reconstruction. Second row: Average of reconstructed intensities.

The speckle coefficient was evaluated for the square indicated in Figure 5, all plots for $C$ in Figure 7 demonstrate improvement with the total number of averaged images, with a tendency for saturation indicating that no significant speckle reduction could be obtained with more images. The values for $C$ are similar between the two optical strategies of plane wave and random checkered phase which use original holograms, however, both are far from the theoretical ideal of $1 / \sqrt{h}$.

To single out the best one of these two optical strategies, we compared the quality on the averaged images. Since the random checkered pattern includes randomly placed phase jumps, the result after the Fourier filtering is a residual amplitude modulation; consequently, the reconstructed images present some darkening in the places of the phase jumps. As we created the patterns to be randomly distributed, the averaged image $\langle I\rangle$ tends to hide these effects, but a minimum degree of deterioration is inevitable. In contrast, the plane waves do not present any degree of amplitude modulation and so the perceived quality of the averaged image is better than the one from the checkered phase strategy.

\subsection{Synthetic Holograms}

We evaluated our numerical strategy to obtain synthetic holograms in combination with the plane wave holograms experimentally recorded. To simplify the analysis, we fixed the number of holograms generated using this strategy to $h_{s}=20$, matching the number $h$ of holograms experimentally recorded. We evaluated the quality of the final reconstruction for different patch sizes $G$. Figure 6 contains the results, which were selected as exact divisions of $2048 \times 2048$, the size of the registered holograms. 
The curves of the speckle coefficient indicate that there is a significant improvement in comparison to the results obtained by the optical strategy, yet not a significant variation among different $G$ values. In contrast, the size of the patch significantly decreases the resolution as it is presented in the profiles plot. For $G=512$, we observe the best resolution since the edges are more defined in comparison to smaller patch sizes, from prior knowledge about the physical attributes of our dice object, we know that the cavity in the center, where the profile crosses, has vertical edges. From Figure 6 we can see that the profile corresponding to $G=512$ presents the more vertical transition from the light region to the dark center. Moreover we can observe that the specular reflection in the center of the cavity of the dice, presents a higher intensity value, indicating a larger efficiency of diffraction in the reconstruction, which implies more fidelity. Therefore, we consider this to be the optimal value.
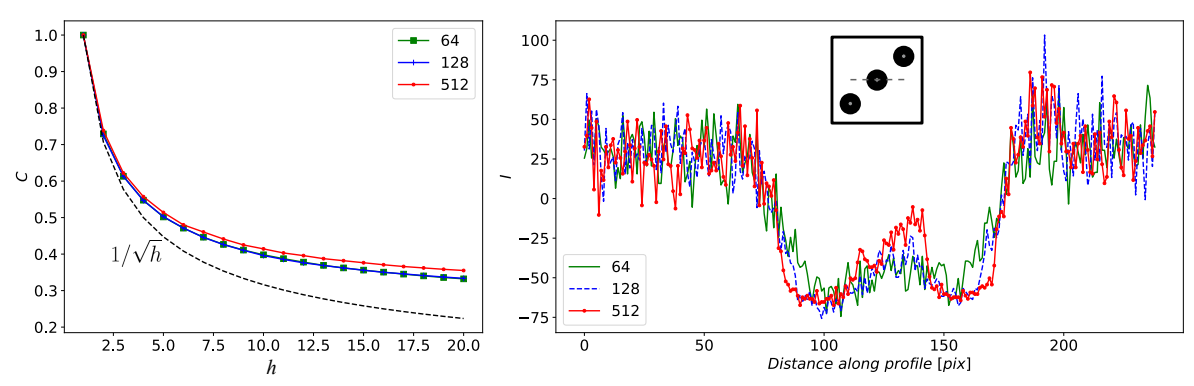

Figure 6. Comparison of speckle reduction and image quality for different cell sizes. (Left): speckle coefficient. (Right): Line profile for the final averaged images corresponding with different values of patch sizes $G$. The profiles have been vertically shifted by subtracting each corresponding mean.

In the third column of Figure 5, we present an individual reconstruction along with the average reconstruction for $G=512$. The individual reconstruction presents an increase in speckle in comparison to the ones obtained by the originally recorded registers. This indicates the random 3D resampling effect. The averaged intensity has a better image quality in comparison to the one obtained by the original holograms. In Figure 7 the correlation coefficient matrix in the third column indicates smaller values, as well as a loss in the regularity that is present in the plane wave strategy, thus further indicating that the new speckle obtained by the resampling is more uncorrelated than the one in the recorded holograms. Similarly, the speckle coefficient plot, clearly demonstrates the improvement gained by the resampling strategy.

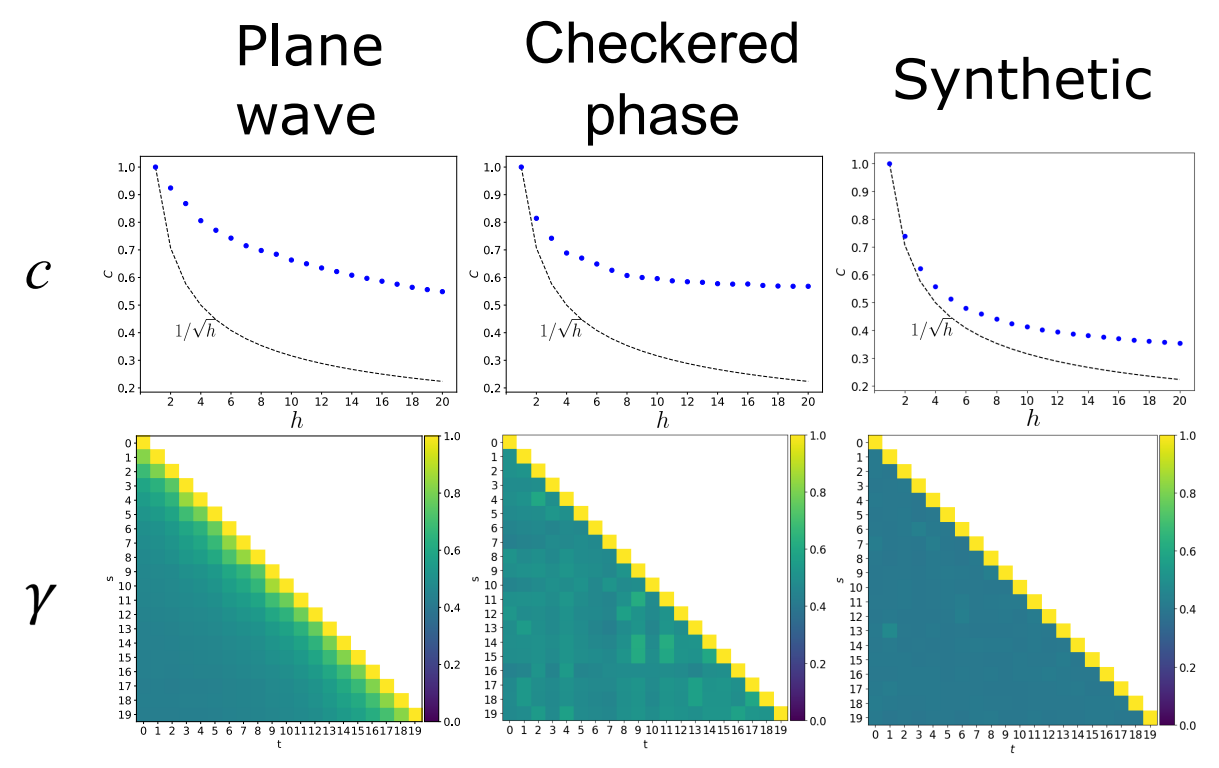

Figure 7. Behavior of the speckle metrics for the three methodologies. First row: speckle coefficient for the square region indicated in Figure 6. Second row: correlation coefficients for every image pair. 


\section{Conclusions}

We presented an alternative methodology to reduce speckle on digital holographic imaging based on a DMD device. The simplicity and robustness of the setup allow us to test different strategies to obtain multiple registers with uncorrelated speckle. We determined that simple plane waves of varying tilt can provide enough variance to improve the quality when multiple reconstructed intensities are averaged. We presented a complementary numerical processing of the registers that allows improving the final reconstruction. A random 3D resampling of the original hologram stack allows obtaining newly composed holograms. The new synthetic holograms are produced from the superposition of nonoverlapping squared patches selectively extracted from the original holograms using binary masks. The parameters of the resampling provide control over the randomness of the synthetic holograms, particularly the patch size being the most important, as it requires to balance the desired increased uncorrelation with the loss of resolution. We determined that the best patch size responds to a factor of 1:1/4 with respect to the original hologram size. Further improvements in our speckle reduction technique can be obtained if the angular variation of the plane waves is increased. With a larger angular range, the recorded holograms will present a larger speckle variance, thus producing a smoother averaged reconstructed intensity. Due to the fast switching nature of the DMD and the computational simplicity of our resampling algorithm, our methodology has potential applicability to fast dynamic scenarios.

Author Contributions: Conceptualization, J.R., N.C.-R. and J.H.-R.; methodology, J.R., N.C.-R. and J.H.-R.; validation, J.R. and J.H.-R.; formal analysis, J.R. and J.H.-R.; investigation J.R. and N.C.-R.; data curation, J.R.; writing — original draft preparation, J.R.; writing—review and editing, J.R. and J.H.-R.; visualization, J.R.; project administration, N.C.-R.; funding acquisition, N.C.-R. All authors have read and agreed to the published version of the manuscript.

Funding: This research was supported by Instituto Tecnologico Metropolitano (Grant number P17217).

Conflicts of Interest: The authors declare no conflicts of interest.The funders had no role in the design of the study; in the collection, analyses, or interpretation of data; in the writing of the manuscript; or in the decision to publish the results.

\section{References}

1. Schnars, U.; Jüptner, W.P.O. Digital recording and numerical reconstruction of holograms. Meas. Sci. Technol. 2002, 13, R85-R101. [CrossRef]

2. Nygate, Y.N.; Singh, G.; Barnea, I.; Shaked, N.T. Simultaneous off-axis multiplexed holography and regular fluorescence microscopy of biological cells. Opt. Lett. 2018, 43, 2587-2590. [CrossRef]

3. Machikhin, A.S.; Polschikova, O.V.; Vlasova, A.G.; Pozhar, V.E. Processing of three-wavelength interference pattern for single-shot quantitative phase imaging. In Proceedings of the Twelfth International Conference on Digital Image Processing (ICDIP 2020), Osaka, Japan, 19-22 May 2020. [CrossRef]

4. Nomura, T.; Okamura, M.; Nitanai, E.; Numata, T. Image quality improvement of digital holography by superposition of reconstructed images obtained by multiple wavelengths. Appl. Opt. 2008, 47, D38-D43. [CrossRef] [PubMed]

5. Rong, L.; Xian, W.; Pan, F.; Liu, S.; Li, R. Speckle noise reduction in digital holography by use of multiple polarization holograms. Chin. Opt. Lett. 2010, 8, 653-655. [CrossRef]

6. Garcia-Sucerquia, J.; Ramírez, J.H.; Castaneda, R. Incoherent recovering of the spatial resolution in digital holography. Opt. Commun. 2006, 62-67. [CrossRef]

7. Baumbach, T.; Kolenovic, E.; Kebbel, V.; Jüptner, W. Improvement of accuracy in digital holography by use of multiple holograms. Appl. Opt. 2006, 45, 6077-6085. [CrossRef]

8. Herrera-Ramirez, J.A.; Hincapie-Zuluaga, D.A.; Garcia-Sucerquia, J. Speckle noise reduction in digital holography by slightly rotating the object. Opt. Eng. 2016, 55, 1-6. [CrossRef]

9. Mori, Y.; Fukuoka, T.; Nomura, T. Speckle reduction in holographic projection by random pixel separation with time multiplexing. Appl. Opt. 2014, 53, 8182-8188. [CrossRef]

10. Garcia-Sucerquia, J.; Ramírez, J.A.H.; Prieto, D.V. Reduction of speckle noise in digital holography by using digital image processing. Optik 2005, 116, 44-48. [CrossRef] 
11. Uzan, A.; Rivenson, Y.; Stern, A. Speckle denoising in digital holography by nonlocal means filtering. Appl. Opt. 2013, 52, A195. [CrossRef]

12. Hincapie, D.; Herrera-Ramírez, J.; Garcia-Sucerquia, J. Single-shot speckle reduction in numerical reconstruction of digitally recorded holograms. Opt. Lett. 2015, 40, 1623. [CrossRef]

13. Leng, J.; Zhou, Z.; Li, F.; Zheng, Q.; Liu, G. Speckle noise suppression using part of pixels in a single-exposure digital hologram. Opt. Eng. 2017, 56, 053103. [CrossRef]

14. Bianco, V.; Paturzo, M.; Memmolo, P.; Finizio, A.; Ferraro, P.; Javidi, B. Random resampling masks: A non-Bayesian one-shot strategy for noise reduction in digital holography. Opt. Lett. 2013, 38, 619-621. [CrossRef]

15. Rong, L.; Xiao, W.; Pan, F. Reduction of speckle noise in digital holography by multiple holograms. Chin. Opt. Lett. 2009, 8, 73823T. [CrossRef]

16. Chenggen, Q.; Xin, K.; Cho-Jui, T. Speckle noise reduction in digital holography by multiple holograms. Opt. Eng. 2007, 46, 115801. [CrossRef]

17. Junmin Leng, J.L.; Xinzhu Sang, X.S.; Binbin Yan, B.Y. Speckle noise reduction in digital holography with spatial light modulator and nonlocal means algorithm. Chin. Opt. Lett. 2014, 12, 040301-40305. [CrossRef]

18. Nesbitt, R.S.; Smith, S.L.; Molnar, R.A.; Benton, S.A. Holographic recording using a digital micromirror device. In Proceedings of the SPIE 3637, Practical Holography XIII, San Jose, CA, USA, 25 March 1999. [CrossRef]

19. Bianco, V.; Memmolo, P.; Paturzo, M.; Finizio, A.; Javidi, B.; Ferraro, P. Quasi noise-free digital holography. Light Sci. Appl. 2016, 5. [CrossRef]

20. Ren, Y.X.; Lu, R.D.; Gong, L. Tailoring light with a digital micromirror device. Ann. Der Phys. 2015, 527, 447-470. [CrossRef]

21. Lee, W.H. III Computer-Generated Holograms: Techniques and Applications. Prog. Opt. 1978, 16, 119-232. [CrossRef]

22. Mirhosseini, M.; na Loaiza, O.S.M.; Chen, C.; Rodenburg, B.; Malik, M.; Boyd, R.W. Rapid generation of light beams carrying orbital angular momentum. Opt. Express 2013, 21, 30196-30203. [CrossRef]

23. Tur, M.; Chin, K.C.; Goodman, J.W. When is speckle noise multiplicative? Appl. Opt. 1982, 21, 1157-1159. [CrossRef]

24. Yang, J.H.X. Fast reduction of speckle noise in real ultrasound images. Signal Process. 2013, 684-694. [CrossRef]

25. Goodman, J.W. Some fundamental properties of speckle. J. Opt. Soc. Am. 1976, 66, 1145-1150. [CrossRef]

26. Goodman, J. Introduction to Fourier Optics; Roberts and Company Publishers:Greenwood Village, CO, USA, 2005.

Publisher's Note: MDPI stays neutral with regard to jurisdictional claims in published maps and institutional affiliations.

(C) 2020 by the authors. Licensee MDPI, Basel, Switzerland. This article is an open access article distributed under the terms and conditions of the Creative Commons Attribution (CC BY) license (http:/ / creativecommons.org/licenses/by/4.0/). 